\title{
(2) OPEN ACCESS \\ Physiological effects of high-flow nasal cannula therapy in preterm infants
}

\author{
Zheyi Liew, ${ }^{1,2}$ Alan C Fenton, ${ }^{3,4}$ Sundeep Harigopal, ${ }^{3,4}$ Saikiran Gopalakaje, \\ Malcolm Brodlie @ (1, ${ }^{1,2}$ Christopher J O'Brien ${ }^{1}$
}

\begin{abstract}
- Additional material is published online only. To view, please visit the journal online (http://dx.doi.org/10.1136/ archdischild-2018-316773)
\end{abstract}

${ }^{1}$ Paediatric Respiratory Medicine, Great North Children's

Hospital, Newcastle upon Tyne Hospitals NHS Foundation Trust,

Newcastle upon Tyne, UK Institute of Cellular Medicine, Newcastle University, Newcastle upon Tyne, UK

${ }^{3}$ Newcastle Neonatal Service, Newcastle upon Tyne Hospitals NHS Foundation Trust, Newcastle upon Tyne, UK ${ }^{4}$ Newcastle University, Newcastle upon Tyne, UK

\section{Correspondence to}

Dr Malcolm Brodlie, Paediatric Respiratory Medicine, Great North Children's Hospital, Newcastle upon Tyne NE1 4LP, UK; m.j.brodlie@ncl.ac.uk

$\mathrm{MB}$ and $\mathrm{CJO} \mathrm{O}^{\prime} \mathrm{B}$ contributed equally.

Received 30 December 2018 Revised 27 April 2019

Accepted 3 May 2019 Published Online First 23 May 2019

\section{ABSTRACT}

Objective High-flow nasal cannula (HFNC) therapy is increasingly used in preterm infants despite a paucity of physiological studies. We aimed to investigate the effects of HFNC on respiratory physiology.

Study design A prospective randomised crossover study was performed enrolling clinically stable preterm infants receiving either HFNC or nasal continuous positive airway pressure (nCPAP). Infants in three current weight groups were studied: <1000 g, 1000-1500 g and >1500 g. Infants were randomised to either first receive HFNC flows 8-2 L/ min and then nCPAP $6 \mathrm{~cm} \mathrm{H}_{2} \mathrm{O}$ or nCPAP first and then HFNC flows 8-2 L/min. Nasopharyngeal end-expiratory airway pressure (pEEP), tidal volume, dead space washout by nasopharyngeal end-expiratory $\mathrm{CO}_{2}(\mathrm{pEECO})_{2}$, oxygen saturation and vital signs were measured.

Results A total of 44 preterm infants, birth weights 500-1900 g, were studied. Increasing flows from 2 to $8 \mathrm{~L} / \mathrm{min}$ significantly increased pEEP (mean $2.3-6.1 \mathrm{~cm}$ $\mathrm{H}_{2} \mathrm{O}$ ) and reduced $\mathrm{pEECO}$ (mean $2.3 \%-0.9 \%$ ). Tidal volume and transcutaneous $\mathrm{CO}_{2}$ were unchanged. Significant differences were seen between pEEP generated in open and closed mouth states across all HFNC flows (difference $0.6-2.3 \mathrm{~cm} \mathrm{\textrm {H } _ { 2 }} \mathrm{O}$ ). Infants weighing $<1000 \mathrm{~g}$ received higher $\mathrm{pEEP}$ at the same HFNC flow than infants weighing $>1000 \mathrm{~g}$. Variability of pEEP generated at HFNC flows of $6-8 \mathrm{~L} / \mathrm{min}$ was greater than nCPAP $\left(2.4-13.5\right.$ vs $\left.3.5-9.9 \mathrm{~cm} \mathrm{H}_{2} \mathrm{O}\right)$.

Conclusions HFNC therapy produces clinically significant pEEP with large variability at higher flow rates. Highest pressures were observed in infants weighing $<1000 \mathrm{~g}$. Flow, weight and mouth position are all important determinants of pressures generated. Reductions in $\mathrm{pEECO}$ support HFNC's role in dead space washout.

\section{INTRODUCTION}

High-flow nasal cannula (HFNC) therapy is increasingly used in preterm infants; perceived benefits include ease of use, increased comfort and bonding. Systematic reviews have concluded that HFNC has similar efficacy to other non-invasive respiratory support in preterm infants $>28$ weeks gestation. ${ }^{23}$ However, as primary support in respiratory distress syndrome, two recent randomised controlled trials found HFNC to be inferior to nasal continuous positive airway pressure (nCPAP). ${ }^{45}$ There is wide variation in the clinical use of HFNC, for example, flow rates and weaning strategies. ${ }^{1}$ This may be partly explained by a lack of understanding of HFNC's mechanisms of action in neonates. ${ }^{6}$

The few physiological studies performed have involved differing flow rates and measurement

\section{What is already known on this topic?}

- High-flow nasal cannula (HFNC) therapy has been rapidly adopted and is increasingly used in preterm infants.

- Mechanisms of action of HFNC are poorly understood; previous studies have found conflicting results, used varied methodology and have included very few infants weighing $<1000 \mathrm{~g}$.

- Reduction of dead space ventilation is thought to be one of the mechanisms of action of HFNC but this has not been demonstrated in preterm infants.

\section{What this study adds?}

We prospectively evaluated the physiological effects of a range of HFNC flow rates from 2 to $8 \mathrm{~L} /$ min in preterm infants, including a substantial number weighing $<1000 \mathrm{~g}$.

- The airway pressure generated during HFNC is dependent on multiple factors, including increasing with flow rate; considerable variability was demonstrated.

- Physiological effects of HFNC include reduction in dead space ventilation, respiratory rate and improved oxygenation.

techniques, small sample sizes and some only in vitro models. ${ }^{7}$ These have produced conflicting conclusions about pressures generated, relationships with infant weight, mouth leak and comparisons with nCPAP ${ }^{8-15}$ Furthermore, the ability of HFNC to wash out airway dead space in infants has been proposed as a major physiological mechanism but not demonstrated in preterm infants. ${ }^{6} 16$ There are minimal data on infants weighing $<1000 \mathrm{~g}$ despite frequent use of flows of up to $8 \mathrm{~L} / \mathrm{min}$ with uncertainty about airway pressures generated. ${ }^{89}$

In this study, we comprehensively evaluated the physiological effects of a range of HFNC flows including airway pressures, dead space washout, tidal volume, minute ventilation and gas exchange, compared with nCPAP $6 \mathrm{~cm} \mathrm{H}_{2} \mathrm{O}$.

\section{METHODS}

Study design

Prospective randomised crossover study in a tertiary neonatal unit (clinical trials.gov NCT02200900 pre-results). Written informed 


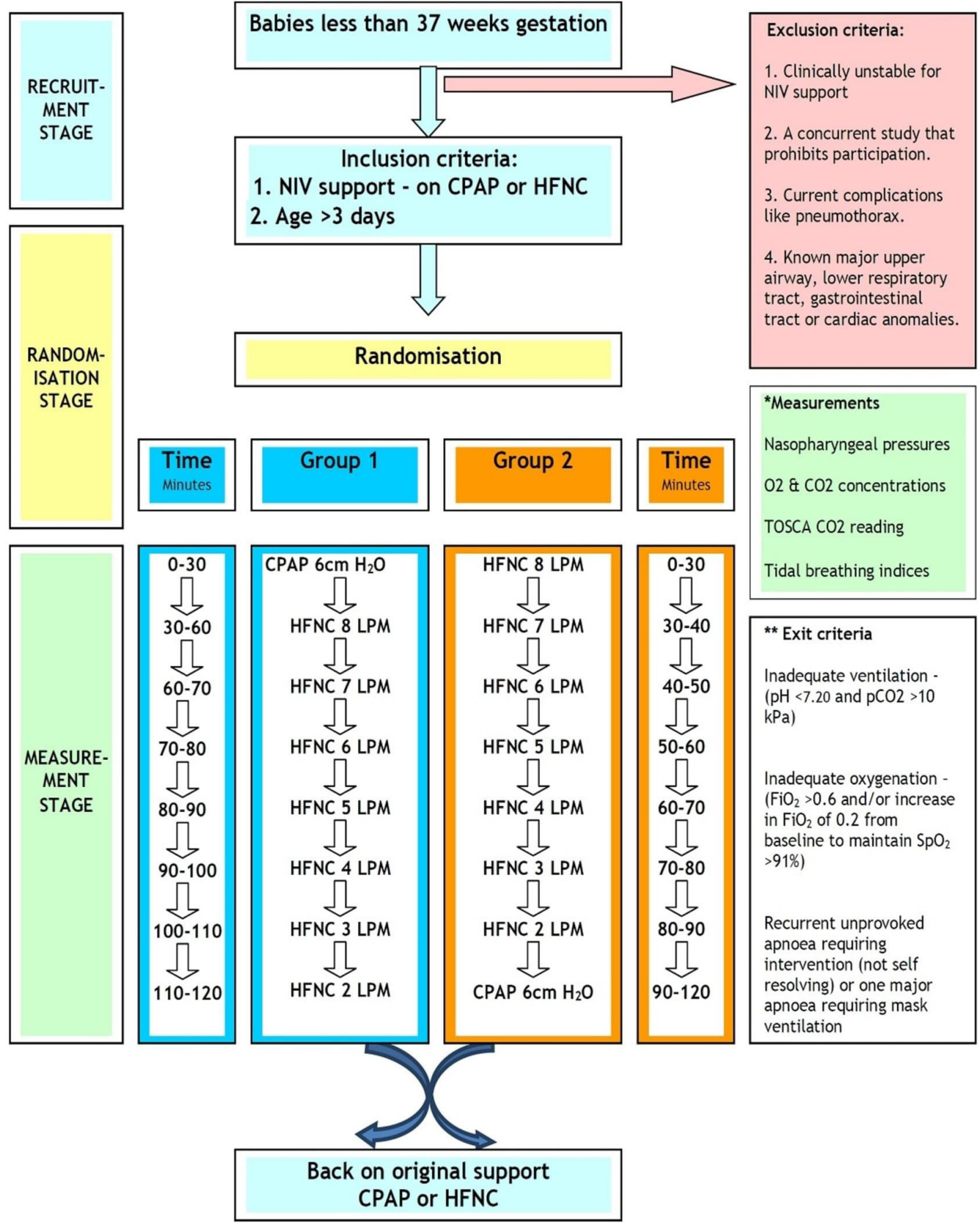

Figure 1 Study flow chart and pathway. Detailed study design and procedures including inclusion, exclusion and exit criteria. CPAP, continuous positive airway pressure; FiO2, oxygen concentration; HFNC, high-flow nasal annula; LPM, litres per minute; NIV, non-invasive ventilation; SpO2, oxygen saturation;TOSCA, transcutaneous $\mathrm{CO2}$.

consent was obtained from parents. A volunteer sample of stable infants $<37$ weeks gestation, aged $>3$ days and receiving $\mathrm{nCPAP}$ or HFNC for the preceding 12 hours were randomised to group 1 (nCPAP then HFNC) or group 2 (HFNC then nCPAP, see figure 1). The study design was developed with Newcastle and North Tyneside Research Ethics Committee (14/NE/0093) to balance acquisition of the best quality data against the potential for destabilisation in this vulnerable patient group. HFNC flows were adjusted and measurements repeated in a set sequence by $1 \mathrm{~L} / \mathrm{min}$ to avoid large pressure changes and destabilisation (figure 1). Measurements during nCPAP were performed at a set pressure of $6 \mathrm{~cm} \mathrm{H}_{2} \mathrm{O}$. The timing of studies was arranged to avoid feeds and were delayed $\geqq 30 \mathrm{~min}$ during transition between modes and at study entry (see online supplementary methods and figure S1).

Study size and statistical analysis

Sample size was calculated with airway pressure as the primary outcome using data from previous studies (Minitab V.17). ${ }^{8} 11$ Infants were stratified into current weight groups $<1000 \mathrm{~g}, 1000$ $1500 \mathrm{~g}$ and $>1500 \mathrm{~g}$. Twelve infants in each group provided adequate sample size to detect a pressure difference of $0.4 \mathrm{~cm}$ $\mathrm{H}_{2} \mathrm{O}$ between flow rates with $80 \%$ power and type 1 error of 0.05 . An additional three infants per group compensated for study dropouts. See online data supplement for statistical tests used. 
Table 1 Characteristics of infants in each weight category

\begin{tabular}{|c|c|c|c|c|c|c|c|c|}
\hline \multirow[b]{2}{*}{ Weight category } & \multicolumn{2}{|c|}{$<1000 \mathrm{~g}(\mathrm{n}=15)$} & \multicolumn{2}{|c|}{$1000-1500 g(n=15)$} & \multicolumn{2}{|c|}{$>1500 \mathrm{~g}(\mathrm{n}=14)$} & \multicolumn{2}{|c|}{ All infants } \\
\hline & Mean & Median (range) & Mean & Median (range) & Mean & Median (range) & Mean & Median (range) \\
\hline Birth gestation (weeks) & 27.0 & $27.6(23.1-30.4)$ & 27.2 & $27.6(23.6-31.1)$ & 26.8 & $26.7(23.3-31.6)$ & 27.0 & $26.9(23.1-31.6)$ \\
\hline Current gestation (weeks) & 30.4 & $30.1(28.3-33.3)$ & 31.7 & $31.6(29.9-34.3)$ & 35.6 & $34.3(31.1-42.1)$ & 32.5 & $31.8(28.3-42.1)$ \\
\hline Age (days) & 26.9 & $15(4-87)$ & 32.9 & $36(3-76)$ & 61.6 & $58(5-132)$ & 40 & $35(3-132)$ \\
\hline Birth weight (g) & 750 & $720(500-1140)$ & 970 & $920(500-1440)$ & 970 & 850 (520-1900) & 890 & $850(500-1900)$ \\
\hline Current weight (g) & 880 & $910(610-1000)$ & 1310 & $1250(1140-1500)$ & 2150 & $1870(1520-4200)$ & 1430 & $1250(610-4200)$ \\
\hline
\end{tabular}

\section{Data sources and measurement}

The Fabian Therapy Evolution (Acutronic Medical) provided HFNC and nCPAP. Nasal prongs (NeoFlow, Armstrong Medical) were fitted and inserted as per manufacturer's recommendation to allow leak around prongs and connected to an AquaVent-Neo breathing circuit (Armstrong Medical) with standard humidification (MR850, Fisher and Paykel). Nasal prongs and diameter of nares were ascertained using a measurement tape. The nCPAP interface used was the IHCA600 (Armstrong Medical) fitted to optimise seal. Humidification was provided during nCPAP using the same humidifier. Nasopharyngeal end-expiratory airway pressure (pEEP) was measured using a suction catheter with two distal side holes (Argyle Gentle Flow 6/8Fr, Covidien) connected to a pressure transducer (B\&D Electromedical, range $0-30 \mathrm{~cm}$ $\mathrm{H}_{2} \mathrm{O}$ ). A $50 \mathrm{~mL} /$ hour microinfuser airflow applied at the catheter inlet avoided occlusion. For details of placement see online supplementary figure S2. Dead space washout was evaluated by measuring nasopharyngeal end-expiratory $\mathrm{CO}_{2}$ concentration $\left(\mathrm{pEECO}_{2}\right)$ using an analyser (AD Instruments) and the same catheter.

As previously described, mouth position was recorded as 'open naturally' or 'closed' (pacifier inserted to create a seal, finger lift under chin or naturally closed) at each HFNC flow rate, but not during nCPAP as the primary focus was airway physiology during HFNC therapy. ${ }^{8}$

Tidal volume changes were measured by electromagnetic inductance plethysmography (VoluSense), previously validated in preterm infants (online supplementary methods). ${ }^{17}$
Transcutaneous $\mathrm{CO}_{2}$ (TOSCA 500 monitor, Radiometer Medical ApS), oxygen saturation and heart rate (Masimo pulse oximeter) were recorded.

Premeasurement transducer and analyser calibration were performed (online supplementary methods). A multichannel recorder (PowerLab, AD Instruments) allowed synchronised recording and graphical presentation of data, applied sampling frequency $100 \mathrm{~Hz}$ (online supplementary figure S3).

\section{Data extraction and analysis}

A $1 \mathrm{~min}$ stabilisation period without data extraction followed each respiratory support adjustment. All artefact-free breaths (each selected block containing $\geq 10$ consecutive breaths, online supplementary figure S4) at each step were analysed.

\section{RESULTS}

\section{Participants}

Forty-eight eligible infants were recruited. Data from the first three infants were not analysed due to technical problems with pEEP measurement technique; results from one infant were unanalysable due to missing data. Table 1 details the characteristics of participants; 27 (61\%) were male. For baseline respiratory support settings see online supplementary table S1.

\section{Generated $\mathrm{pEEP}$ at different HFNC flow rates}

Table 2 shows pEEP generated at each level of support. There was a positive correlation between $\mathrm{pEEP}$ and flow rate $\left(\mathrm{r}_{\mathrm{s}}=0.589\right.$,

Table 2 pEEP at each respiratory support level including effect of mouth position

\begin{tabular}{|c|c|c|c|c|c|c|c|c|}
\hline & HFNC & & & & & & & nCPAP \\
\hline Flow (L/min) & 2 & 3 & 4 & 5 & 6 & 7 & 8 & $6 \mathrm{~cm} \mathrm{H}_{2} \mathrm{O}$ \\
\hline pEEP $\left(\mathrm{cm} \mathrm{H}_{2} \mathrm{O}\right)$ & $2.3 \pm 1.3$ & $3.4 \pm 1.6$ & $4.1 \pm 1.6$ & $4.2 \pm 1.4$ & $4.8 \pm 1.7$ & $5.4 \pm 2.0$ & $6.1 \pm 2.1$ & $6.4 \pm 1.5$ \\
\hline Mouth closed & 2.7 & 4.0 & 4.8 & 5.1 & 5.7 & 6.4 & 7.3 & $\mathrm{n} / \mathrm{a}$ \\
\hline Mouth open & 2.1 & 2.9 & 3.3 & 3.5 & 4.2 & 4.5 & 5.1 & $\mathrm{n} / \mathrm{a}$ \\
\hline Difference & 0.6 & 1.1 & 1.5 & 1.6 & 1.4 & 1.9 & 2.3 & $\mathrm{n} / \mathrm{a}$ \\
\hline$P$ value* & 0.002 & 0.0001 & 0.0001 & 0.0001 & 0.0001 & 0.0001 & 0.0001 & $\mathrm{n} / \mathrm{a}$ \\
\hline $\mathrm{pEECO}_{2}(\%)$ & $2.3 \pm 1.6$ & $1.9 \pm 1.5$ & $1.7 \pm 1.5$ & $1.7 \pm 1.7$ & $1.4 \pm 1.5$ & $1.0 \pm 1.3$ & $0.9 \pm 1.1$ & $2.4 \pm 1.8$ \\
\hline $\mathrm{Vt} / \mathrm{kg}(\mathrm{mL} / \mathrm{kg})$ & $4.3 \pm 1.9$ & $3.8 \pm 2.0$ & $4.0 \pm 1.9$ & $4.4 \pm 2.3$ & $3.9 \pm 1.6$ & $3.9 \pm 1.6$ & $4.2 \pm 1.8$ & $4.7 \pm 2.1$ \\
\hline $\mathrm{RR}(\mathrm{bpm}) \dagger$ & $70 \pm 17$ & $64 \pm 15$ & $66 \pm 18$ & $64 \pm 17$ & $63 \pm 18$ & $61 \pm 16$ & $62 \pm 15$ & $66 \pm 17$ \\
\hline MV (mL/kg/min) & $309 \pm 162$ & $235 \pm 122$ & $258 \pm 128$ & $269 \pm 157$ & $247 \pm 133$ & $239 \pm 99$ & $268 \pm 148$ & $315 \pm 176$ \\
\hline $\mathrm{TCCO}_{2}(\mathrm{kPa})$ & $6.2 \pm 1.1$ & $6.2 \pm 0.8$ & $6.1 \pm 0.9$ & $6.1 \pm 1.1$ & $6.1 \pm 1.0$ & $6.3 \pm 1.0$ & $6.3 \pm 0.9$ & $6.5 \pm 1.1$ \\
\hline $\mathrm{S}_{\mathrm{p}} \mathrm{O}_{2}(\%) \ddagger$ & $92.0 \pm 4.4$ & $93.5 \pm 3.8$ & $94.2 \pm 4.0$ & $94.8 \pm 3.5$ & $95.3 \pm 3.0$ & $95.9 \pm 3.2$ & $96.4 \pm 3.3$ & $95.1 \pm 3.8$ \\
\hline HR (bpm) & $156 \pm 13$ & $158 \pm 12$ & $159 \pm 12$ & $160 \pm 12$ & $160 \pm 10$ & $162 \pm 12$ & $164 \pm 12$ & $165 \pm 13$ \\
\hline
\end{tabular}

Effects of HFNC therapy on pEECO ${ }_{2}$, tidal volume, ventilation, gas exchange and haemodynamics.

Expressed as means \pm SD.

*Wilcoxon signed rank test (mouth position).

tAnalysis of variance, $\mathrm{p}=0.047$, when HFNC $8 \mathrm{~L} / \mathrm{min}$ reduced to HFNC $2 \mathrm{~L} / \mathrm{min}$ across all flows.

$\neq$ Friedman, $\mathrm{p} \leq 0.0001$, when HFNC $8 \mathrm{~L} / \mathrm{min}$ reduced to HFNC $2 \mathrm{~L} / \mathrm{min}$ across all flows.

$\mathrm{HR}$, heart rate; HFNC, high-flow nasal cannula; n/a, not available; nCPAP, nasal continuous positive airway pressure; MV, minute vol; pEEP, nasopharyngeal end-expiratory pressure; pEECO2, nasopharyngeal end-expiratory $\mathrm{CO} 2$; RR, respiratory rate; Sp02, oxygen saturation; TCCO2, transcutaneous $\mathrm{CO2}$; Vt, tidal volume. 


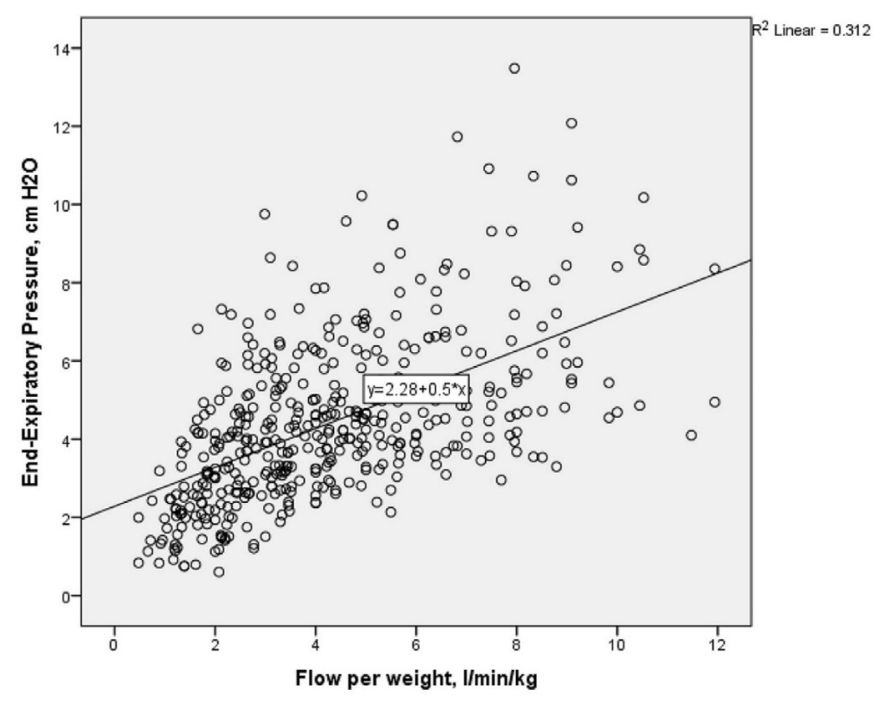

Figure 2 Scatter plot of relationship between nasopharyngeal endexpiratory positive pressure (pEEP) and weight-adjusted flow rate. Figure demonstrates large variability of pEEP measured above $6 \mathrm{~L} / \mathrm{min} /$ $\mathrm{kg}$, with some pEEP measured up to $8-13 \mathrm{~cm} \mathrm{H}_{2} \mathrm{O}$.

$\mathrm{p}<0.0001)$. On average, $\mathrm{pEEP}$ increased by $0.6 \mathrm{~cm} \mathrm{H}_{2} \mathrm{O}$ for each $1 \mathrm{~L} / \mathrm{min}$ flow rate increment in HFNC $\left(\mathrm{R}^{2}=0.311,95 \%\right.$ CI 0.47 to 0.61 ). Figure 2 shows variability in pEEP generated, especially at higher flows. The SD and range of pEEP generated at flows $>6 \mathrm{~L} / \mathrm{min}$ was greater than nCPAP $6 \mathrm{~cm} \mathrm{H}_{2} \mathrm{O}$ (range 2.4-13.5 compared with $3.5-9.9 \mathrm{~cm} \mathrm{H}_{2} \mathrm{O}$ ).

\section{Effect of mouth position on HFNC}

Generated pEEP was influenced by mouth position, being significantly higher (difference $0.6-2.3 \mathrm{~cm} \mathrm{H}_{2} \mathrm{O}, \mathrm{p}<0.05$ ) with mouth closed, across all flow rates (table 2).

\section{Effect of weight}

Weight was negatively correlated $\left(\mathrm{r}_{\mathrm{s}}=-0.247, \mathrm{p}<0.0001\right)$ with pEEP; on average decreasing by $0.7 \mathrm{~cm} \mathrm{H}_{2} \mathrm{O}(95 \% \mathrm{CI}-0.9$ to $-0.3, \mathrm{p}<0.0001)$ for each $\mathrm{kg}$ increase. Table 3 demonstrates the pEEP received by infants in each weight category. Overall, pEEP generated was higher in smaller infants at all flows compared with larger infants (pEEP received in $1000 \mathrm{~g}$ group $>1000$ $1500 \mathrm{~g}>1500 \mathrm{~g})$. Generated pEEP reached $8-13 \mathrm{~cm} \mathrm{H}_{2} \mathrm{O}$ at higher flows in some infants (figure 2).

\section{Effect of prong-to-nares ratio}

pEEP and prong-to-nares ratio were positively correlated $\left(r_{s}=0.165, p<0.0001\right)$. These ratios were further divided into high-leak and low-leak groups $(<0.7$ and $>0.7)$. Generated pEEP was statistically significantly higher in the low-leak compared with the high-leak group at flows $2-4 \mathrm{~L} / \mathrm{min}(\mathrm{p}<0.05$, online supplementary figure S5). We consistently observed a drop in pEEP generated if the nasal prongs became partially dislodged during measurements.

\begin{tabular}{|c|c|c|c|c|c|}
\hline \multirow[b]{2}{*}{ HFNC flow rate (L/min) } & \multirow[b]{2}{*}{ Weight category (g) } & \multicolumn{2}{|l|}{ pEEP } & \multicolumn{2}{|l|}{$\mathrm{pEECO}_{2}$} \\
\hline & & Mean \pm SD & P value* & Mean \pm SD & $P$ valuet \\
\hline \multirow[t]{3}{*}{2} & $<1000$ & $3.0 \pm 1.6$ & 0.021 & $1.6 \pm 1.3$ & 0.014 \\
\hline & $1000-1500$ & $2.3 \pm 1.2$ & & $2.2 \pm 1.7$ & \\
\hline & $>1500$ & $1.8 \pm 0.7$ & & $3.2 \pm 1.5$ & \\
\hline \multirow[t]{3}{*}{3} & $<1000$ & $4.2 \pm 1.9$ & 0.005 & $1.10 \pm 0.99$ & 0.003 \\
\hline & $1000-1500$ & $3.2 \pm 1.5$ & & $1.73 \pm 1.54$ & \\
\hline & $>1500$ & $2.6 \pm 0.6$ & & $2.96 \pm 1.41$ & \\
\hline \multirow[t]{3}{*}{4} & $<1000$ & $5.0 \pm 1.9$ & 0.005 & $0.6 \pm 0.6$ & 0.001 \\
\hline & $1000-1500$ & $3.6 \pm 1.3$ & & $2.0 \pm 1.6$ & \\
\hline & $>1500$ & $3.4 \pm 0.9$ & & $2.5 \pm 1.4$ & \\
\hline \multirow[t]{3}{*}{5} & $<1000$ & $4.6 \pm 1.5$ & NS & $0.7 \pm 0.7$ & 0.002 \\
\hline & $1000-1500$ & $4.0 \pm 1.2$ & & $2.0 \pm 2.1$ & \\
\hline & $>1500$ & $3.9 \pm 1.6$ & & $2.5 \pm 1.6$ & \\
\hline \multirow[t]{3}{*}{6} & $<1000$ & $5.5 \pm 2.2$ & NS & $0.5 \pm 0.8$ & $<0.0001$ \\
\hline & $1000-1500$ & $4.4 \pm 1.2$ & & $1.3 \pm 1.5$ & \\
\hline & $>1500$ & $4.5 \pm 1.3$ & & $2.2 \pm 1.5$ & \\
\hline \multirow[t]{3}{*}{7} & $<1000$ & $5.9 \pm 2.5$ & NS & $0.2 \pm 0.3$ & $<0.0001$ \\
\hline & $1000-1500$ & $5.1 \pm 1.4$ & & $1.1 \pm 1.6$ & \\
\hline & $>1500$ & $5.1 \pm 1.8$ & & $1.9 \pm 1.5$ & \\
\hline \multirow[t]{3}{*}{8} & $<1000$ & $6.6 \pm 2.5$ & NS & $0.2 \pm 0.4$ & $<0.0001$ \\
\hline & $1000-1500$ & $6.0 \pm 2.0$ & & $1.2 \pm 2.1$ & \\
\hline & $>1500$ & $5.8 \pm 1.8$ & & $1.8 \pm 1.5$ & \\
\hline
\end{tabular}

Infants weighing $<1000 \mathrm{~g}=15,1000-1500 \mathrm{~g} \mathrm{n}=15,>1500 \mathrm{~g} \mathrm{n}=14$.

Expressed in means $\pm S D$.

*Jonckheere-Terpstra test for ordered alternatives showed that there was a statistically significant trend of higher pEEP in infants weighing $<1000 \mathrm{~g}$ compared with infants $1000-1500 \mathrm{~g}$ and/or $>1500 \mathrm{~g}$ at flows $2-4 \mathrm{~L} / \mathrm{min}$.

†Jonckheere-Terpstra test for ordered alternatives showed that there was a statistically significant trend of lower $\mathrm{pEECO}_{2}$ in infants weighing $<1000 \mathrm{~g}$ compared with larger weight groups infants $1000-1500 \mathrm{~g}$ and/or $>1500 \mathrm{~g}$ across all flows.

NS, non-significant. 


\section{Analysis of factors that affect pEEP generated}

On multiple linear regression flow rate, mouth position, current weight and gestation but not prong-to-nares ratio significantly predicted $\mathrm{pEEP}$ and account for a significant amount of its variance $(\mathrm{F}(4431)=143.768, \mathrm{p}<0.0001), \mathrm{R}^{2}=0.572, \mathrm{R}^{2}=\mathrm{ad}-$ justed 0.568 ). Flow rate was the most significant independent variable, followed by mouth position, weight and current gestation. Predicted pEEP generated $=-6.373+0.525 \times(-$ flow rate, $\mathrm{L} / \mathrm{min})+1.454 \times($ mouth position, $0=$ open and $1=$ closed $)-1.856 \times($ weight $\quad(\mathrm{kg}))+0.307 \times($ current gestation (weeks)).

\section{Comparison of $p E E P$ generated by HFNC versus nCPAP}

Mean pEEP with nCPAP $6 \mathrm{~cm} \mathrm{H}_{2} \mathrm{O}$ across all weight groups was $6.4 \mathrm{~cm} \mathrm{H} \mathrm{H}_{2} \mathrm{O}(95 \% \mathrm{CI} 6.0$ to 6.7$)$; higher than HFNC 2-7 L/min $(\mathrm{p}<0.05)$ and comparable to HFNC $8 \mathrm{~L} / \mathrm{min}$. However, specifically in infants weighing $<1000 \mathrm{~g}$, the mean pEEP with nCPAP

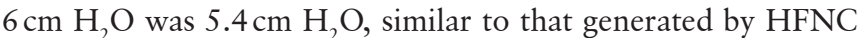
in the 4-6L/min range but statistically higher than with HFNC at flows of $2-3 \mathrm{~L} / \mathrm{min}$. Importantly, in infants weighing $<1000 \mathrm{~g}$ pEEP generated by HFNC $7-8 \mathrm{~L} / \mathrm{min}$ was higher than nCPAP $6 \mathrm{~cm} \mathrm{H}_{2} \mathrm{O}$.

\section{Dead space washout effect}

Despite a clear pressure respiratory waveform, confirmed catheter patency and satisfactory position, $\mathrm{pEECO}_{2}$ was often markedly attenuated at higher flows, supporting a significant washout effect. There was a strong, negative correlation between $\mathrm{pEECO}_{2}$ and weight-corrected flow rate $\left(r_{s}=-0.323, p<0.0001\right)$. Open mouth state was associated with greater washout effect (lowered $\mathrm{pEECO}_{2}$ measured during mouth open), especially at high flow rates though was not statistically significant (online supplementary table S2). Current weight and $\mathrm{pEECO}_{2}$ were positively correlated $\left(\mathrm{r}_{\mathrm{s}}=0.484, \mathrm{p}<0.0001\right)$. The reduction of $\mathrm{pEECO}_{2}$ was greatest in infants weighing $<1000 \mathrm{~g}$, and was statistically significant compared with the other 2 weight groups (table 3 ). The mean nCPAP $\mathrm{pEECO}_{2}$ was $2.4 \%$ and was higher than HFNC across all flows, but only achieved significance at $6-8 \mathrm{~L} /$ $\min (\mathrm{p}<0.05)$.

\section{Effects of HFNC on tidal volume, ventilation and gas exchange}

Reduction of HFNC from 8 to $2 \mathrm{~L} / \mathrm{min}$ did not result in a change of weight-corrected tidal volume despite significant reduction in pEEP (table 2). Minute volume increased when flows reduced. Reducing flows from 8 to $2 \mathrm{~L} / \mathrm{min}$ statistically significantly increased respiratory rate $(\mathrm{p}=0.047)$ and significantly lowered $\mathrm{S}_{\mathrm{p}} \mathrm{O}_{2}$ by $4.4 \%(\mathrm{p}<0.0001)$. Each $1 \mathrm{~L} / \mathrm{min}$ flow rate increment improved $\mathrm{S}_{\mathrm{p}} \mathrm{O}_{2}$ by $0.6 \%$. Importantly, 13 subjects (30\%) required $\mathrm{FiO}_{2}$ increased by $2 \%-9 \%$ when flows reduced from 8 to $2 \mathrm{~L} /$ min (eight were $<1000 \mathrm{~g}$, three were $1000-1500 \mathrm{~g}$ and two were $>1500 \mathrm{~g}$ ). $\mathrm{TCCO}_{2}$ was unchanged. Comparing nCPAP $6 \mathrm{~cm} \mathrm{H}_{2} \mathrm{O}$ with HFNC $8 \mathrm{~L} / \mathrm{min}$ at equal generated pEEP, HFNC $8 \mathrm{~L} / \mathrm{min}$ resulted in similar weight-corrected tidal volume, $\mathrm{TCCO}_{2}, \mathrm{SpO}_{2}$ and heart rate (all $\mathrm{p}>0.05$ ).

\section{DISCUSSION}

Key findings of our study were that flow rate was linearly related to pressure delivered, as suggested previously, ${ }^{8-11} 141518$ and that weight, age, mouth position and prong-to-nares ratio are significant factors in determining pressure delivered. A substantial number of infants weighing $<1000 \mathrm{~g}$, in whom there is a paucity of previous data, were included. Furthermore, unlike previous studies, ${ }^{8-12} 141519$ we included flow rates of $2-8 \mathrm{~L} / \mathrm{min}$ that are commonly prescribed clinically. ${ }^{1}$ Previous data on pressures generated during HFNC are conflicting, likely due to different measurement techniques, small sample sizes and narrow flow rate protocols. ${ }^{8-12141519}$

Across all infants studied HFNC $8 \mathrm{~L} / \mathrm{min}$ was comparable to $6 \mathrm{~cm} \mathrm{H} \mathrm{H}_{2} \mathrm{O} \mathrm{nCPAP} \mathrm{but} \mathrm{average} \mathrm{pEEP} \mathrm{generated} \mathrm{by} \mathrm{HFNC} \mathrm{of} 6 \mathrm{~L} /$ min was lower than that generated by $\mathrm{CPAP} 6 \mathrm{~cm} \mathrm{H}_{2} \mathrm{O}$, which may be relevant to the recent finding in randomised studies that HFNC is inferior to nCPAP when used as primary support for preterm infants with respiratory distress syndrome. ${ }^{45}$ We also found considerable variability in pEEP generated at higher HFNC flows and at any given flow rate, the smallest infants received significantly higher pressures. Increased understanding of the mechanisms of action of HFNC in preterm infants should inform the design of future high-quality clinical studies. ${ }^{20} 21$

In our study, pEEP with the mouth closed was significantly higher than mouth open across all flow rates, similar to the findings of Arora et al in older infants with bronchiolitis. ${ }^{14}$ Previous neonatal studies have varied in results from no pressure generated when mouth open ${ }^{9}$ to no effect ${ }^{8}$ with work in an in vitro model ${ }^{10}$ showing that a leak as low as $30 \%$ leads to a dramatic reduction in pressure. Although not part of the study protocol, we observed that $\mathrm{pEEP}$ measurements were consistently lower when prongs were accidentally loosened highlighting the importance of correct positioning as per manufacturer's instructions.

Generated pEEP correlated negatively with infants' weight, a finding similar to some studies ${ }^{81822}$ but not all. ${ }^{13-15}$ Importantly, 30 of our subjects were $<1500 \mathrm{~g}$, with $15<1000 \mathrm{~g}$. Some of the generated pEEPs $\left(8-13 \mathrm{~cm} \mathrm{H}_{2} \mathrm{O}\right)$ at higher flow rates were higher than those generated by $6 \mathrm{~cm} \mathrm{H}_{2} \mathrm{O}$ nCPAP, contrasting with observations by Lavizzari et al, ${ }^{19}$ where only $75 \%$ of infants reached pEEP of $4 \mathrm{~cm} \mathrm{H}_{2} \mathrm{O}$ and rarely $>5 \mathrm{~cm}$ $\mathrm{H}_{2} \mathrm{O}$. This may be due to our larger number of small infants and higher flow rates $(>6 \mathrm{~L} / \mathrm{min})$. In infants weighing $<1000 \mathrm{~g}$, we found that flows as low as $4-6 \mathrm{~L} / \mathrm{min}$ generate average pEEP similar to nCPAP $6 \mathrm{~cm} \mathrm{H} \mathrm{H}_{2} \mathrm{O}$ and flows of 7-8 $\mathrm{L} / \mathrm{min}$ delivered

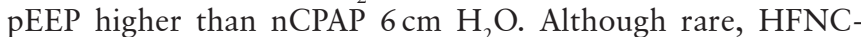
related complications have been reported. ${ }^{23}{ }^{24}$ Awareness of pressures delivered to vulnerable infants is important and may aid clinicians in prescribing flow rates. A recent survey found that $66 \%$ of clinicians adjusted flow in increments of $0.5-1 \mathrm{~L} /$ min when weaning: our data suggest that flow changes of $0.5 \mathrm{~L} / \mathrm{min}$ are unlikely to have a major impact on respiratory parameters. ${ }^{1}$

Washout of nasopharyngeal dead space thereby increasing alveolar ventilation and improving $\mathrm{CO}_{2}$ elimination has been suggested as a mechanism of action of HFNC. ${ }^{6}$ This has been investigated in in vitro models,,$^{25}$ an animal study ${ }^{16}$ and adults $^{2728}$ but not in preterm infants. We found that increasing flows from 2 to $8 \mathrm{~L} / \mathrm{min}$ led to significant reductions in $\mathrm{pEECO}_{2}$ and decreases in minute ventilation probably due to reductions in dead space ventilation, with the greatest effects seen in the smallest infants but without a significant change in $\mathrm{TCCO}_{2}$. Möller et al also demonstrated that dead space washout was flow-dependent, ${ }^{26}$ and reduction of $\mathrm{CO}_{2}$ rebreathing occurred during HFNC in tracheostomised adults. ${ }^{27}$ The $\mathrm{pEECO}_{2}$ was

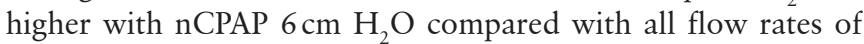
HFNC supporting the hypothesis that HFNC reduces dead space better than nCPAP, similar to recent in vitro findings that washout times for nCPAP were significantly longer than HFNC by $16.2 \%{ }^{25}$ Our observation that mouth open was associated with lower $\mathrm{pEECO}_{2}$ measured compared with mouth closed was similar to previous work, ${ }^{25}$ suggesting that the shorter oral 
pathway surpasses the nasal route by providing the majority of the washout effect.

We acknowledge that the design of our study in vulnerable infants balanced patient safety as our overriding concern against acquisition of the best quality data possible in terms of invasiveness of measurements and timing at each level of respiratory support. There are significant limitations to the use of $\mathrm{TCCO}_{2}$ in premature infants, ${ }^{29}$ but it is non-invasive and arterial blood gas measurements would have been impractical. Our finding of a lack of change of $\mathrm{TCCO}_{2}$, which was within the normal range, during HFNC was similar to previous reports. ${ }^{283031}$ We have also only investigated one HFNC and nCPAP delivery system.

The weight-corrected tidal volume measured across 2-8 L/ min of flow and on nCPAP did not differ significantly, similar to previous reports. ${ }^{19} 3032$ Explanations could be variability of sleep state in our infants as ventilatory responses to HFNC are different during wakefulness and sleep, ${ }^{33}$ and variability in infants' need for non-invasive support at the time of study and age range. Increases in pEEP result in increases in functional residual capacity while tidal volume in infants may be more dependent on the degree of lung disease and work of breathing. Mauri et al recently demonstrated in adults that HFNC increases end-expiratory lung volume, but tidal volume was unchanged. ${ }^{32}$

We demonstrated that reducing flows from 8 to $2 \mathrm{~L} / \mathrm{min}$ led to a significant increase in respiratory rate, in agreement with previous studies. ${ }^{1015} 19$ Interestingly, we found that both respiratory and heart rate were generally higher during nCPAP therapy, possibly explained by better tolerance of HFNC. Increasing flows improved oxygenation saturation, as demonstrated previously. ${ }^{18}$

Although all infants tolerated the study protocol well, with no adverse events, $30 \%$ of participants (highest in the $<1000 \mathrm{~g}$ group) required an oxygen increment to maintain their $\mathrm{SaO}_{2}$ within set parameters, which could have mitigated changes in some parameters but was essential to ensure safety. Without simultaneous oesophageal pressure measurement, we could not investigate compliance and work of breathing. However, adding this would have entailed significant additional handling, and an oesophageal pressure probe may have impacted on airway physiology and caused discomfort. Although the nasopharyngeal catheter used to measure pressure was similar to a nasogastric feeding tube, it is conceivable that it generated a degree of leak. However, HFNC apparatus are designed as 'leaky systems' to prevent barotrauma and the CPAP system used compensates automatically to maintain a set pressure.

In summary, multiple factors impact the pEEP delivered by HFNC in preterm infants, which leads to considerable variability. Extremely small infants are at greatest risk of receiving high pEEP. Physiological effects of increasing HFNC flow rate include raised airway pressure, improved oxygenation, lower respiratory rate and improved effective alveolar ventilation by reducing dead space ventilation.

Acknowledgements The authors would like to thank all of the families who gave consent for their infants to take part in this study. The authors are also indebted to all of the nursing and medical staff of Ward 35, Royal Victoria Infirmary, Newcastle upon Tyne Hospitals NHS Foundation Trust who have supported this work. The authors would also like to thank Kim Pearce for statistical advice to ZL via Newcastle University Faculty of Medical Sciences Graduate School.

Contributors ZL and SG: enrolled infants, performed measurements, collected and analysed data. ACF, SH, MB and CJO'B: conceived the study, developed the protocol and study design and analysed the data. All authors had intellectual input to the manuscript and approved the final version as submitted and agree to be accountable for all aspects of the work.

Funding Funding from Newcastle upon Tyne Hospitals NHS Charity for equipment. MB was supported by a Medical Research Council Clinician Scientist Fellowship (MR/M008797/1). The research was supported by the National Institute for Health Research Newcastle Biomedical Research Centre based at Newcastle Hospitals NHS Foundation Trust and Newcastle University.

Disclaimer The views expressed are those of the author(s) and not necessarily those of the NHS, the NIHR or the Department of Health.

Competing interests $\mathrm{ZL}, \mathrm{ACF}, \mathrm{SH}, \mathrm{SG}$ and $\mathrm{CJO} \mathrm{O}^{\prime} \mathrm{B}$ : none. MB: not related to this work: investigator-led research grants from Pfizer and Roche Diagnostics; speaker fees paid to Newcastle University from Novartis, Roche Diagnostics and TEVA. Travel expenses to educational meeting Boehringer Ingelheim and Vertex Pharmaceuticals.

Patient consent for publication Not required.

Provenance and peer review Not commissioned; externally peer reviewed.

Data sharing statement Data available on reasonable request to ORCiD 00000003-4591-8299 once study is fully published and analysed subject to approval by full study team.

Open access This is an open access article distributed in accordance with the Creative Commons Attribution 4.0 Unported (CC BY 4.0) license, which permits others to copy, redistribute, remix, transform and build upon this work for any purpose, provided the original work is properly cited, a link to the licence is given, and indication of whether changes were made. See: https://creativecommons.org/ licenses/by/4.0/.

\section{ORCID iD}

Malcolm Brodlie http://orcid.org/0000-0003-4591-8299

\section{REFERENCES}

1 Shetty S, Sundaresan A, Hunt K, et al. Changes in the use of humidified high flow nasal cannula oxygen. Arch Dis Child Fetal Neonatal Ed 2016;101:F371-2.

2 Wilkinson D, Andersen C, O'Donnell CP, et al. High flow nasal cannula for respiratory support in preterm infants. Cochrane Database Syst Rev 2016;2:CD006405.

3 Kotecha SJ, Adappa R, Gupta N, et al. Safety and efficacy of high-flow nasal cannula therapy in preterm infants: a meta-analysis. Pediatrics 2015;136:542-53.

4 Roberts CT, Owen LS, Manley BJ, et al. Nasal high-flow therapy for primary respiratory support in preterm infants. N Engl J Med 2016;375:1142-51.

5 Murki S, Singh J, Khant C, et al. High-Flow nasal cannula versus nasal continuous positive airway pressure for primary respiratory support in preterm infants with respiratory distress: a randomized controlled trial. Neonatology 2018;113:235-41.

6 Dysart K, Miller TL, Wolfson MR, et al. Research in high flow therapy: mechanisms of action. Respir Med 2009;103:1400-5

7 Shetty S, Greenough A. Review finds insufficient evidence to support the routine use of heated, humidified high-flow nasal cannula use in neonates. Acta Paediatr 2014;103:898-903.

8 Wilkinson DJ, Andersen CC, Smith K, et al. Pharyngeal pressure with high-flow nasal cannulae in premature infants. J Perinatol 2008:28:42-7.

9 Kubicka ZJ, Limauro J, Darnall RA. Heated, humidified high-flow nasal cannula therapy: yet another way to deliver continuous positive airway pressure? Pediatrics 2008; 121:82-8.

10 Lampland AL, Plumm B, Meyers PA, et al. Observational study of humidified high-flow nasal cannula compared with nasal continuous positive airway pressure. J Pediatr 2009;154:177-82.

11 Spence KL, Murphy D, Kilian C, et al. High-flow nasal cannula as a device to provide continuous positive airway pressure in infants. J Perinatol 2007;27:772-5.

12 Saslow JG, Aghai ZH, Nakhla TA, et al. Work of breathing using high-flow nasal cannula in preterm infants. J Perinatol 2006;26:476-80

13 lyer NP, Mhanna MJ. Assessment of end expiratory esophageal pressures during noninvasive respiratory support in very low birth weight (VLBW) infants. American Journal of Respiratory and Critical Care Medicine Conference: American Thoracic Society International Conference, ATS 2014:189.

14 Arora B, Mahajan P, Zidan MA, et al. Nasopharyngeal airway pressures in bronchiolitis patients treated with high-flow nasal cannula oxygen therapy. Pediatr Emerg Care 2012;28:1179-84.

15 Milési C, Baleine J, Matecki S, et al. Is treatment with a high flow nasal cannula effective in acute viral bronchiolitis? A physiologic study1170 Note: Combonie, Gilles [corrected to Cambonie, Gilles]]. Intensive Care MedIntensive Care Medicine 2013;3939:1088-94.

16 Frizzola M, Miller TL, Rodriguez ME, et al. High-flow nasal cannula: impact on oxygenation and ventilation in an acute lung injury model. Pediatr Pulmonol 2011;46:67-74. 
17 Bentsen MH, Eriksen M, Olsen MS, et al. Electromagnetic inductance plethysmography is well suited to measure tidal breathing in infants. ERJ Open Res 2016;2:00062-2016

18 Spentzas T, Minarik M, Patters AB, et al. Children with respiratory distress treated with high-flow nasal cannula. J Intensive Care Med 2009;24:323-8.

19 Lavizzari A, Veneroni C, Colnaghi M, et al. Respiratory mechanics during NCPAP and HHHFNC at equal distending pressures. Arch Dis Child Fetal Neonatal Ed 2014:99:F315-F320.

20 Taha DK, Kornhauser M, Greenspan JS, et al. High Flow Nasal Cannula Use Is Associated with Increased Morbidity and Length of Hospitalization in Extremely Low Birth Weight Infants. J Pediatr 2016;173:50-5.

21 Roberts CT, Owen LS, Davis PG, et al. Chicken or egg? Dangers in the interpretation of retrospective studies. J Pediatr 2016;178:309.

22 Sreenan C, Lemke RP, Hudson-Mason A, et al. High-flow nasal cannulae in the management of apnea of prematurity: a comparison with conventional nasal continuous positive airway pressure. Pediatrics 2001;107:1081-3.

23 Iglesias-Deus A, Pérez-Muñuzuri A, López-Suárez 0, et al. Tension pneumocephalus induced by high-flow nasal cannula ventilation in a neonate. Arch Dis Child Fetal Neonatal Ed 2017:102:F173-5.

24 Jasin LR, Kern S, Thompson S, et al. Subcutaneous scalp emphysema, pneumo-orbitis and pneumocephalus in a neonate on high humidity high flow nasal cannula. $J$ Perinatol 2008:28:779-81.
25 Sivieri EM, Foglia EE, Abbasi S. Carbon dioxide washout during high flow nasal cannula versus nasal CPAP support: An in vitro study. Pediatr Pulmonol 2017; $52: 792-8$.

26 Möller W, Celik G, Feng $S$, et al. Nasal high flow clears anatomical dead space in upper airway models. J App/ Physiol 2015;118:1525-32.

27 Möller W, Feng S, Domanski U, et al. Nasal high flow reduces dead space. J Appl Physiol 2017;122:191-7.

28 Biselli P, Fricke K, Grote L, et al. Reductions in dead space ventilation with nasal high flow depend on physiological dead space volume: metabolic hood measurements during sleep in patients with COPD and controls. Eur Respir J 2018:51:1702251.

29 Janaillac M, Labarinas S, Pfister RE, et al. Accuracy of transcutaneous carbon dioxide measurement in premature infants. Crit Care Res Pract 2016;2016:1-5.

30 Delorme M, Bouchard PA, Simon M, et al. Effects of high-flow nasal cannula on the work of breathing in patients recovering from acute respiratory failure. Crit Care Med 2017:45:1981-8.

31 Biselli PJ, Kirkness JP, Grote L, et al. Nasal high-flow therapy reduces work of breathing compared with oxygen during sleep in COPD and smoking controls: a prospective observational study. J App/ Physio/ 2017;122:82-8.

32 Mauri T, Turrini C, Eronia N, et al. Physiologic effects of high-flow nasal cannula in acute hypoxemic respiratory failure. Am J Respir Crit Care Med 2017;195:1207-15.

33 Mündel T, Feng $S$, Tatkov $S$, et al. Mechanisms of nasal high flow on ventilation during wakefulness and sleep. J App/ Physiol 2013;114:1058-65. 